\title{
Epithelized Free Autograft-A Case Report Series with Different Healing Outcomes
}

\author{
Dr. Khushboo Goel ${ }^{1}$ \\ ${ }^{I}$ MDS, Assistant Professor, Department of Periodontology and Oral Implantology, College of Dental Surgery, \\ B.P Koirala Institute of Health and Sciences, Dharan, Sunsari, Nepal,
}

\begin{abstract}
The gain in gingival soft tissue volume for multifaceted dynamics requires long term stability of gingival margin and interdental papilla height. Four patients presented with Miller's class II and III recession on mandibular incisors. Abundant plaque and calculus, an aberrant frenum and inadequate width of attached gingiva $(A G)$ were localized in the area with complaint of difficulty in cleaning the root surface. Patients underwent epithelized free autograft (Free Gingival Graft) for gain in AG, vestibular deepening and root coverage using direct technique. Healing in 18 months to two years showed creation of thick wide zones of AG with partial root coverage. This provided room for plaque control and halted disease progression. There was greater patient satisfaction as soft tissue and its existence was more important than quantitative level of root coverage. Autografts were cost effective and offered long term predictability. However, esthetic results obtained were different in all four patients. Therefore, this case series addresses various factors that interplay in healing of graft. Further, it highlights that position of fully erupted teeth and inclination of roots along with vascularity of recipient bed are likely to play an essential role that requires further well controlled trials to aid in better esthetic outcome and case selection.
\end{abstract}

Keywords: Autologous, Dental esthetics, Gingiva, Tissue grafts, Transplantation, Wound healing

\section{Introduction}

Adequacy of attached gingiva and its relation to preventing gingival inflammation and apical displacement of soft tissue margin are the debatable issue since the past. At a recent time, the surgical intervention for correction of the inadequate width of attached gingiva also reports contradictory opinion. Series of well-designed experiments and longitudinal study proved that the attached gingiva and its width, have little role in maintaining periodontal health $[1,2]$. Inflammation plays a key role as the main future predictor of attachment loss and therapy should be directed at controlling bacterial plaque and reducing inflammation and there is no need to treat minimally attached gingiva. However, in some patients treatment might be required as the same condition may lead to exposure of an unacceptable amount of root surface, fear of losing a tooth if the patient is observant of receding gums and an inability to effectively perform plaque control in localized area. A study done by Lang \& Loe at al [3]reported that plaque-free areas with $1 \mathrm{~mm}$ or more of attached gingiva were inflamed despite effective oral hygiene and recently in a long term study done for 18 to 35 years found that sites treated with gingival augmentation procedures experience a decrease in attachment loss in contrast to untreated sites [4].

Regeneration of lost gingival tissues is an achievable goal today and of the several techniques available to increase the width of attached gingiva (AG), epithelized free autograft (Free Gingival Graft, FGG) is widely used since it was first described by Bjorn in 1963 [5]. Various factors interplay for the healing events that affect treatment outcome that varies from graft thickness, stabilization of graft, depth of vestibule, wound contraction/shrinkage and atraumatic surgical technique [6].

We report here the treatment of four cases that underwent FGG procedure and the decision to treat was based on persistent gingival inflammation, a shallow vestibular depth in localized area and an aberrant frenum that restricted access for effective oral hygiene [7]. The primary objective of the treatment was to prevent progression and stabilize the band of attached gingiva. There was also an attempt to cover denuded root surface to prevent a recurrent recession by altering gingival thickness and to evaluate factors that may have an effect on the healing of recipient site. Based on visual analog scale (VAS), the patient reported esthetic outcomes that are the soft tissue color and texture were documented. Similarly, the expectations of patients were also evaluated on whether the graft per se conveys any greater resistance to further attachment loss and thus help in the maintenance of oral hygiene at 18 months to two years follow-up.

\section{Pre-Surgical Management}

The patients were apparently well with no significant medical and dental histories. There was no history of any adverse habits. Pre-operative clinical photographs were taken. Intraoral clinical examination revealed gingival inflammation, recession and inadequate width of attached gingiva localized to the mandibular 
central incisors with overall satisfactory oral hygiene. Thick fibrous frenum pull was evident which destabilized the marginal gingiva on movement. The width of keratinized tissue (KT), AG and gingival recession (GR) was recorded with University of North Carolina-15 periodontal probe (Hu-Friedy). The junction of the attached and movable tissue was determined by rolling the alveolar mucosa coronally with the side of a probe. In cases where more than one tooth was treated, the area with least amount of KT was selected. Patients had normal biotype with scalloped gingiva and thickness of the gingiva ranged from least $1.5 \mathrm{~mm}$ to maximum $2 \mathrm{~mm}$ when measured with the direct method [8]. All surgical procedures, pre-operative and post-operative clinical evaluations were done by the author. Root surfaces were mechanically prepared with ultrasonic or hand instruments prior to the mucogingival procedure to allow biological attachment of the grafted tissue to it. Surgery was performed only when satisfactory plaque control was achieved.

\section{Surgery}

A written informed consent was obtained from each patient. The surgical procedure in the cases was similar [9]. The surgical site was anesthetized, soft tissue involving muscle and frenal insertions were removed by sharp dissection to ensure that the graft lies passively with no movement occurring during the function. Recipient site was prepared using a splint-thickness flap with and horizontal incision at the level of cemento-enamel junction extending from the line angle of the adjacent teeth on either side of the recession and two vertical incisions extending well into the alveolar mucosa.

Donor site: The Thickness of palatal tissue was assessed using an endodontic reamer after local anesthesia. Tin-foil templates were used to measure the size of the defect to be filled. These templates were transferred to the palate as a guide to the graft size. To fulfill the objectives of increasing zones of attached gingiva and root coverage procedures, a graft of approximately $1-1.5 \mathrm{~mm}$ of uniform thickness was removed. Care was taken to avoid the palatine blood vessels. The graft obtained was finely inspected for any glandular or fatty tissue remnants. The harvested graft was then placed on the recipient site at the level of interdental papilla height of central incisors for adequate blood supply. No deliberate attempt was made to cover completely cover the root surface area. The graft was sutured by means of horizontal stabilizing sutures and interrupted 4-0 silk sutures. Good adaptation of the flap to the underlying tissues was ensured for adequate diffusion and vitality of tissues. A periodontal pack was placed to protect the surgical site and for the donor site, an acrylic palatal stent was made. An area of graft size with $1.5 \mathrm{~mm}$ depth for placement of gel foam was made on the stent.

Postoperative instructions were given to the patients and were instructed to report to the department after 24 hours and after ten days. Patients were advised to use $0.2 \%$ chlorhexidine solution rinse twice a day during this period. Capsule Amoxicillin 500mg three times a day for 5days and Tablet ibuprofen 400mg two times daily for 3 days were prescribed. At 10 days the palatal wound healing was satisfactory. Visual analog scale (VAS) was used to evaluate patient reported esthetic outcome and expectations based on texture, color, maintenance of oral hygiene, the stability of gingival margins and overall satisfaction in the postoperative period at 18 months to 2 years follow-up. A $10 \mathrm{~cm}$ VAS with 'unacceptable' at the left with score '0' and 'pleased' at the right end with score ' 10 ' as verbal end points was prepared. Forms were given to patients at their follow-up visits (Table 3 ).

\section{Case I}

A 21-year-old female patient reported with the chief complaints of receding gums and difficulty in cleaning the root surface area for one year. The case was diagnosed with Miller's class II GR with an aberrant frenal pull (Fig.1a) (Table 1). After scaling and control of inflammation, an FGG was planned (Fig.1b). After initial preparation (Fig.1c) graft was inlayed on a vascular recipient bed (Fig.1d). At 10 days, the healing was uneventful at both surgical sites but it showed no gain in gingival soft tissue at recipient site (Fig. 1e) and after that, the patient lost to follow-up. However after 18 months, patient self reported to the department (Fig. 1f). There was no deposition of calculus in the localized area with apparently healthy and stable gingival margins. The partial gain in root coverage was achieved through creeping attachment and good color match with adjacent gingiva was observed (Table 2) (Table 3).

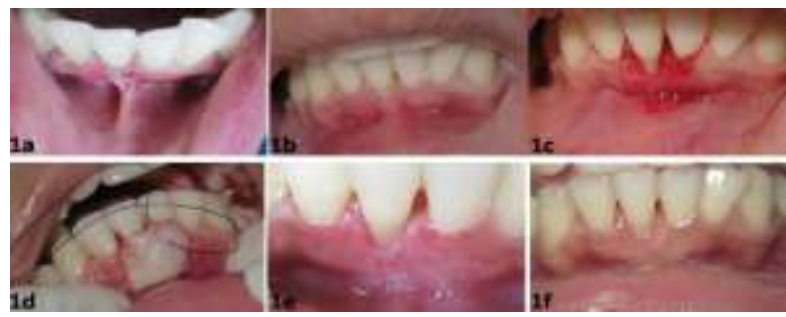

Figure 1: 1a Localized deposit of plaque and calculus with aberrant frenum, 1b: After scaling and root planing, 1c: Recipient bed, 1d: Adaptation of free epithelized graft, 1e: After 10 days, 1f: 18 months follow-up 


\section{Case II}

A 25-year-old female patient reported with the chief complaint of long teeth since 2 years and fear of losing the tooth since 1 month. The case was diagnosed with Millers class III GR and had an aberrant frenal pull (Fig. 2a) (Table 1). Clinically, the recipient bed appeared less vascular and roots seemed prominent (Fig. 2b). After 10 days healing was uneventful (Fig. 2c). At two years follow-up there was sufficient gain in soft tissue volume with the decrease in gingival marginal inflammation (Table 2). An increase in the amount of horizontal as well as vertical shrinkage with a patch like an appearance of the newly gained tissue was also observed that compromised the esthetics with adjacent gingival tissue (Fig. 2d) (Table 3).

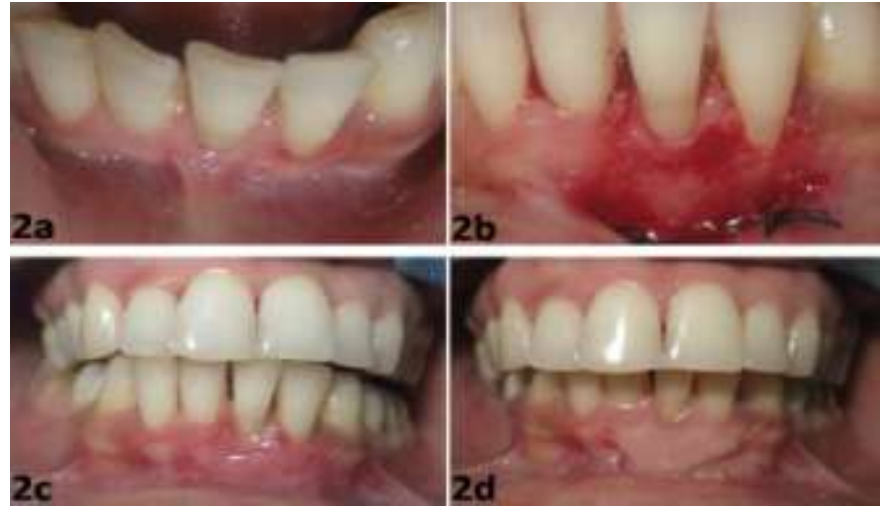

Figure 2: 2a. After scaling and root planing and aberrant frenum, $2 \mathrm{~b}$ : Recipient bed preparation $2 \mathrm{c}$ : After 10 days, $2 \mathrm{~d}$ : 2 years follow-up

\section{Case III}

A 19-year-old male patient reported with the chief complaint of difficulty in cleaning the lower root surface area and long teeth since 6 months (Fig 3a) (Table 1). The case was diagnosed with Miller's class III GR and frenum pull (Fig. 3b). Vascularity of the tissue was prominent during recipient site preparation (Fig. 3c). Two weeks after treatment, a gain in gingival soft tissue was appreciable (Fig. 3d, 3e) (Table 2). At 9 months follow-up, there was no deposition of calculus in the localized area with apparently healthy and stable gingival margins and a good color match (Fig. 3f) (Table 3). The patient had an open bite and was advised for orthodontic consultation but the patient was reluctant due to his financial status.
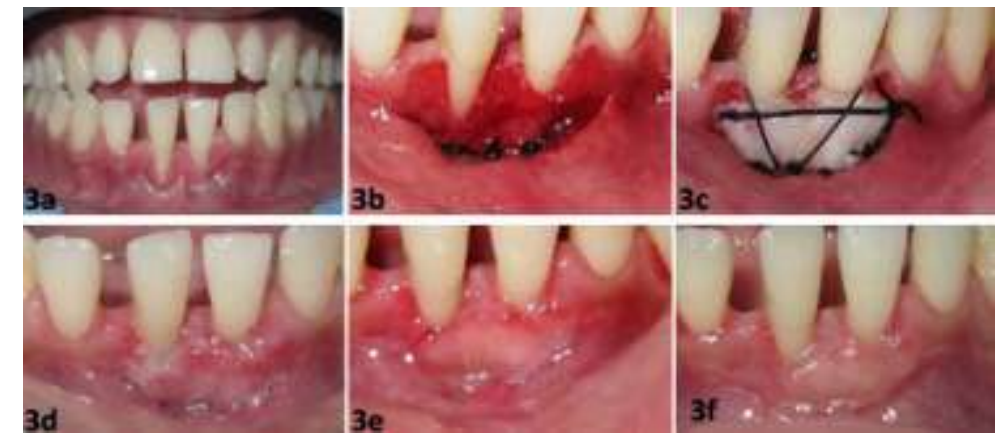

Figure 3: 3a After scaling and root planing with aberrant frenum pull, 3b: Recipient bed preparation, 3c: Free gingival graft with sutures, 3d: After 10 days, 3e: 3 month follow-up, 3f: 9 months follow-up.

\section{Case IV}

A 25-year-old female patient reported with the chief complaint of mobile teeth since 2 years and fear of losing the tooth since 6 months. The case was diagnosed with Miller's class III GR with aberrant frenum pull (Fig. 4a, b) (Table 1). During the preparation of recipient site, as the teeth were positioned labial, less surface area was available and the site appeared less vascular. The depth of the vestibule was inadequate. After 10 days healing was uneventful and there was a gain in soft tissue (Fig. 4c) (Table 2). At 2 year follow-up, there was an increase in width of AG (Fig. 4d). However, appreciable horizontal and vertical shrinkage was observed with the patch like appearance of the tissues with no gain in root coverage. The gingiva was inflamed when the patient presented and maintenance of oral hygiene was not adequate. The patient was satisfied overall as there was a decrease in mobility and some gain in gingival soft tissue (Table 3).The patient was reinforced to maintain oral hygiene. 

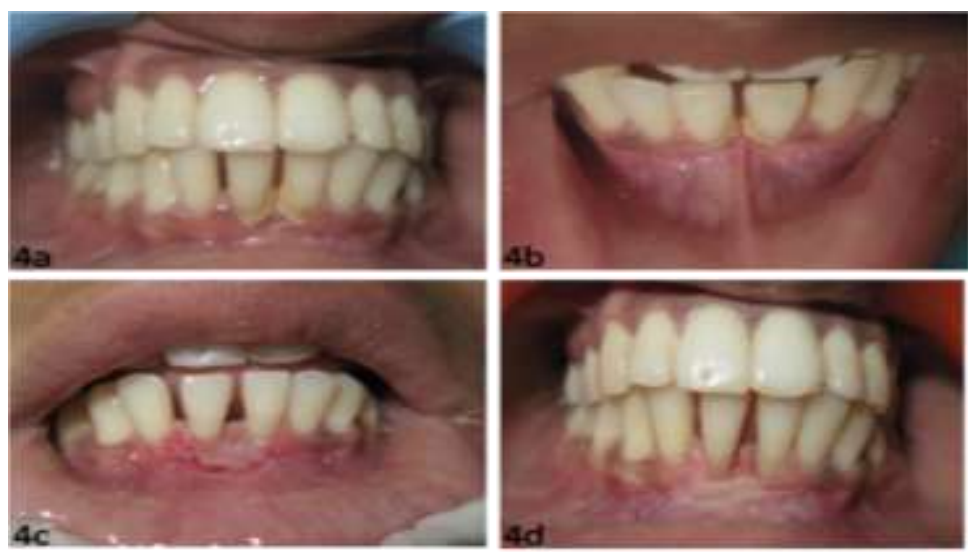

Figure 4: 4a. Initial clinical presentation, 4b: Aberrant frenum pull, 4c: After 10 days, 4d: 2 years follow-up

\section{Discussion}

Transplantation of epithelized free autograft (Free gingival graft, FGG) is one of the most dependable procedures available for all of the above mentioned objectives [6]. The high predictability of this technique in humans has been well documented in histological, cytological and clinical studies [10]. Its autogenous character, ease of technique, cost effectiveness and together with the possibility of implementation in a group of teeth leads FGG to be accepted as the golden standard to increase attached gingiva dimensions [11]. There are several other techniques tried and amongst them, Acellular Dermal Matrix (ADM) allograft is emerging for its advantage of avoiding palatal complications $[12,13]$. However, comparative studies evaluating ADM and free gingival graft show that ADM allograft exhibits more shrinkage and are less effective and predictable than an autogenous FGG $[12,14]$. In this case series, all the patients underwent FGG procedure. The procedure was highly predictable in halting of the disease progression at 18 months to two years follow-up with a wider band of attached gingiva of 2-3mm comparable with other studies. The oral hygiene performance was relatively better for all patients except in case IV. Healing and the final esthetic outcome was, however different. There are various factors that affect the healing period of a free gingival graft. The most critical factor is the blood supply between free gingival graft and recipient site. Other factors include graft shrinkage, the thickness of the graft obtained from the palate, gingival biotype and suturing technique. In all four cases, similar surgical procedure, atraumatic graft stabilization for plasmatic circulation and suture technique was applied.

In the present case series, none of the patients showed any shrinkage at 10 days. There was no shrinkage in case I at 18 months and minimal in case III at 9 months follow-up. However, at two years followup considerable amount of shrinkage resulted in both vertical and horizontal dimensions in case II and case IV. Various amounts of graft shrinkage during the healing period have been reported which ranges between $12 \%$ and 48\% [15-17]. Studies show that shrinkage occurs during the first year after surgery and the width of keratinized tissue remains stable after that $[18,19]$. The results of the case series have demonstrated changes at an individual level. It is speculated that graft thickness might have a significant role in shrinkage. Thicker grafts are difficult to adapt and show more secondary contraction whereas thin grafts are susceptible to increase primary contraction [20]. To minimize the differences in shrinkage during healing, an adequate and standard graft thickness including palatal epithelium, lamina propria and a thin layer of submucosa of 1$1.5 \mathrm{~mm}$ was considered to achieve all objectives. An effort was made to avoid deeper wound in the palate and subsequently to reduce post-operative discomfort. Similarly, tissue biotype can act as a significant factor that influences the esthetic treatment outcomes. Claffey and Shanley [21] noticed thin gingival tissue biotype to show more gingival recession than the thicker biotype and even the thin gingival biotypes to exhibit more shrinkage [17]. Case II and IV had biotype measurement of $1.5 \mathrm{~mm}$ that was more on the thinner aspect which might be one of the reasons for maximum shrinkage in these cases. However, according to Fu et al [22] the underlying bone has a moderate association with the thickness of the labial gingival tissue. Therefore, it is not always necessary that thin gingival tissues have thin underlying bone and effect esthetic treatment outcome.

There was partial root coverage gained in all cases that ranged from 1 to $3 \mathrm{~mm}$ which was approximately 50\%. This proves the high predictability of the technique even in Millers class III gingival recession. Creeping attachment was also observed in case I and II ranging from $0.5 \mathrm{~mm}$ to $1 \mathrm{~mm}$. Creeping attachment is apparently best observed on mandibular anterior teeth with narrow recessions [23, 24]. This phenomenon can be detected 1 to 12 months after graft surgery with an average coverage of about $1 \mathrm{~mm}$. A long term stability of gingival margin was obtained in all cases at two years and it might be speculated that other factors might play a role in healing in FGG to affect the esthetic outcome. An adequately vascularized recipient bed appears to be an important factor in determining functional and esthetic outcomes [25]. In all our cases, the vascular bed was prepared by enhancing local blood flow. Clinically in case I and III, the recipient 
bed was more vascular which might have resulted in better esthetics, though not evaluated histologically. Frenotomy procedure has also been hypothesized to increase vascularization and improve esthetics, however in our cases; it did not seem to aid in improving esthetics.

The position of fully erupted teeth and inclination of roots might also be some of the factors to apparently affect vascularity and hence the healing and esthetic outcome in FGG. In case II the roots seemed prominent and teeth were positioned labially in case IV. Though there was no fenestration or dehiscence encountered during the recipient bed preparation. These factors have been evaluated less in clinical trials which might have influenced the esthetic outcome in our cases.

The patient plays a more important role in determining success in aesthetic procedures and in the acceptance of the postsurgical outcome [26]. Only one patient presented with pain symptom at donor site at day 3 that subsided completely by day 10 . There was no bleeding from the donor as well as recipient site after surgery and the healing was uneventful. A Visual analog scale (VAS) was used that gave an average score of 7.1 can be considered good.

Table 1: Pre-surgical assessment

\begin{tabular}{|c|c|c|c|}
\hline Case & Keratinized Tissue (KT) & Attached Gingiva (AG) & Gingival Recession (GR) \\
\hline Case I & 0 & 0 & $3 \mathrm{~mm}$ \\
\hline Case II & 1 & 0 & $4 \mathrm{~mm}$ \\
\hline Case III & 1 & 0 & $6 \mathrm{~mm}$ \\
\hline Case IV & 0 & 0 & 3 \\
\hline
\end{tabular}

Table 2: Post-surgical assessment

\begin{tabular}{|c|c|c|c|}
\hline Case & Keratinized Tissue (KT) & Attached Gingiva (AG) & Root Coverage (RC) \\
\hline Case I & 3 & 2.5 & $2 \mathrm{~mm}$ \\
\hline Case II & 4 & 3 & $1.5 \mathrm{~mm}$ \\
\hline Case III & 4 & 3 & 3 \\
\hline Case IV & 2 & 1 & 1 \\
\hline
\end{tabular}

Table 3: Evaluation of patient reported esthetic outcome and expectations based on VAS score.

\begin{tabular}{|c|c|c|c|c|c|c|}
\hline Case & Texture & Color & $\begin{array}{c}\text { Maintenance of } \\
\text { Oral Hygiene }\end{array}$ & $\begin{array}{c}\text { Stability of gingival } \\
\text { margins }\end{array}$ & $\begin{array}{c}\text { Overall } \\
\text { satisfaction }\end{array}$ & Average score \\
\hline Case I & 8 & 9 & 9 & 9 & 9 & 8.8 \\
\hline Case II & 5 & 5 & 8 & 8 & 6 & 6.4 \\
\hline Case III & 7 & 8 & 8 & 7 & 8 & 7.6 \\
\hline Case IV & 5 & 6 & 4 & 6 & 7 & 5.6 \\
\hline
\end{tabular}

\section{Conclusion}

Free gingival graft technique is a versatile and predictable procedure. Esthetic results can vary depending on tissue characteristics proper of each patient. There is little information available as to whether tooth position (buccal or lingual), root prominence, and vascularity of recipient bed might influence the outcome of mucogingival procedures. Various factors still need exploration and controlled trials to determine the factors that affect healing of FGG. These can act as a decisive factor for choosing the procedure as well as manage patient's expectations.

\section{Acknowledgements}

The author would like to thank Professor Dr. Shaili Pradhan and Professor Dr. Shivalal Sharma for their constant guidance in all these cases. They both served as scientific advisors and critically reviewed the case series.

\section{Authorship Credit}

The corresponding author is the guarantor of submission.

Khushboo Goel - Substantial contributions to conception and design, Acquisition of data, Analysis and interpretation of data, drafting the article.

The author is accountable for all aspects of the work in ensuring that questions related to the accuracy or integrity of any part of the work are appropriately investigated and resolved.

Disclosure Policy

The author declares that there is no conflict of interest regarding the publication of this paper. 


\section{References}

[1]. Kennedy JE, Bird WC, Palcanis KG, Dorfman HS. A longitudinal evaluation of varying widths of attached gingiva. J Clin Periodontol 1985;12:667-675.

[2]. Wennstrom J, Lindhe J. Role of attached gingiva for maintenance of periodontal health. Healing following excisional and grafting procedures in dogs. J Clin Periodontol 1983;10:206-21.

[3]. Lang NP, Loe H. The relationship between the width of keratinized gingiva and gingival health. J Periodontol 1972;43:623-7.

[4]. Agudio G, Cortellini P, Buti J, Pini Prato G. Periodontal Conditions of Sites Treated With Gingival Augmentation Surgery Compared With Untreated Contralateral Homologous Sites: An 18- to 35-Year Long-Term Study. J Periodontol 2016;87:13711378.

[5]. Bjorn H. Free transplantation of gingiva propria (abstract). In: Symposium in Periodontology in Malmo. Odontologisk Revy $1963 ; 14: 321-323$.

[6]. Sullivan HC, Atkins JH. Free autogenous gingival grafts. I. Principles of successful grafting. Periodontics 1968;6:121-9.

[7]. Merijohn GK. Management and prevention of gingival recession. Periodontol 2000. 2016;71:228-242

[8]. Greenberg J, Laster L, Listgarten MA. Transgingival probing as a potential estimator of alveolar bone level J Periodontol. 1976;47:514-517.

[9]. Miller PD, Jr. Root coverage using the free soft tissue autograft following citric acid application. III. A successful and predictable procedure in areas of deep-wide recession. Int J Periodontics Restorative Dent 1985;5:14-37.

[10]. Gordon HP, Sullivan HC, Atkins JH. Free autogenous gingival grafts. II. Supplemental findings--histology of the graft site. Periodontics 1968;6:130-3

[11]. Karring T, Ostergaard E, Loe H. Conservation of tissue specificity after heterotopic transplantation of gingiva and alveolar mucosa. J Periodontal Res 1971;6:282-293.

[12]. Wei PC, Laurell L, Geivelis M, Lingen MW, Maddalozzo D. Acellular dermal matrix allografts to achieve increased attached gingiva. Part 1. A clinical study. J Periodontol 2000;71:1297-1305.

[13]. Scarano A, Barros RR, Iezzi G, Piattelli A, Novaes AB, Jr. Acellular dermal matrix graft for gingival augmentation: a preliminary clinical, histologic, and ultrastructural evaluation. J Periodontol 2009;80:253-259.

[14]. Agarwal C, Tarun Kumar AB, Mehta DS. Comparative evaluation of free gingival graft and AlloDerm((R)) in enhancing the width of attached gingival: A clinical study. Contemp Clin Dent 2015;6:483-488.

[15]. James WC, McFall WT, Jr. Placement of free gingival grafts on denuded alveolar bone. Part I: clinical evaluations. J Periodontol 1978;49:283-290.

[16]. Silva CO, Ribeiro Edel P, Sallum AW, Tatakis DN. Free gingival grafts: graft shrinkage and donor-site healing in smokers and nonsmokers. J Periodontol 2010;81:692-701.

[17]. Hatipoglu H, Keceli HG, Guncu GN, Sengun D, Tozum TF. Vertical and horizontal dimensional evaluation of free gingival grafts in the anterior mandible: a case report series. Clin Oral Investig 2007;11:107-113.

[18]. Orsini M, Orsini G, Benlloch D, Aranda JJ, Lazaro P, Sanz M. Esthetic and dimensional evaluation of free connective tissue grafts in prosthetically treated patients: a 1-year clinical study. J Periodontol 2004;75:470-477.

[19]. Rateitschak KH, Egli U, Fringeli G. Recession: a 4-year longitudinal study after free gingival grafts. J Clin Periodontol 1979;6:158164.

[20]. Mormann W, Schaer F, Firestone AR. The relationship between success of free gingival grafts and transplant thickness. Revascularization and shrinkage--a one year clinical study. J Periodontol 1981;52:74-80.

[21]. Claffey N, Shanley D. Relationship of gingival thickness and bleeding to loss of probing attachment in shallow sites following nonsurgical periodontal therapy. J Clin Periodontol 1986;13:654-657.

[22]. Fu JH, Yeh CY, Chan HL, Tatarakis N, Leong DJ, Wang HL. Tissue biotype and its relation to the underlying bone morphology. J Periodontol 2010;81:569-574.

[23]. Matter J, Cimasoni G. Creeping attachment after free gingival grafts. J Periodontol 1976;47:574-579.

[24]. Matter J. Creeping attachment of free gingival grafts. A five-year follow-up study. J Periodontol 1980;51:681-685.

[25]. Ducic Y, Pontius AT, Smith JE. Lipotransfer as an adjunct in head and neck reconstruction. Laryngoscope 2003;113:1600-1604.

[26]. Camargo PM, Melnick PR, Kenney EB. The use of free gingival grafts for aesthetic purposes. Periodontol 2000 2001;27:72-96. 